\title{
Chemical composition and some biological activities of the essential oils from basil Ocimum different cultivars
}

\author{
Arpi Avetisyan ${ }^{1}$, Anahit Markosian¹, Margarit Petrosyan², Naira Sahakyan², Anush Babayan², Samvel Aloyan \\ and Armen Trchounian ${ }^{2 *}$
}

\begin{abstract}
Background: The plants belonging to the Ocimum genus of the Lamiaceae family are considered to be a rich source of essential oils which have expressed biological activity and use in different area of human activity. There is a great variety of chemotypes within the same basil species. Essential oils from three different cultivars of basil, O. basilicum var. purpureum, $O$. basilicum var. thyrsiflora, and O. citriodorum Vis. were the subjects of our investigations.
\end{abstract}

Methods: The oils were obtained by steam distillation in a Clevenger-type apparatus. The gas chromatography mass selective analysis was used to determine their chemical composition. The antioxidant activities of these essential oils were measured using 1,1-diphenyl-2-picrylhydrazyl assays; the tyrosinase inhibition abilities of the given group of oils were also assessed spectophotometrically, and the antimicrobial activity of the essential oils was determined by the agar diffusion method, minimal inhibitory concentrations were expressed.

Results: According to the results, the qualitative and quantitative composition of essential oils was quite different: O. basilicum var. purpureum essential oil contained $57.3 \%$ methyl-chavicol (estragol); O. basilicum var. thyrsiflora oil had $68.0 \%$ linalool. The main constituents of 0 . citriodorum oil were nerol (23.0\%) and citral (20.7\%). The highest antioxidant activity was demonstrated by 0 . basilicum var. thyrsiflora essential oil. This oil has also exhibited the highest tyrosinase inhibition level, whereas the oil from O. citriodorum cultivar demonstrated the highest antimicrobial activity.

Conclusions: The results obtained indicate that these essential oils have antioxidant, antibacterial and antifungal activity and can be used as natural antioxidant and antimicrobial agents in medicine, food industry and cosmetics.

Keywords: Ocimum, Essential oil, Methyl-chavicol, Linalool, Nerol, Citral, Antioxidant, Antibacterial activity

\section{Background}

The plants belonging to the basil genome or Ocimum genus of the Lamiaceae family are aromatic ones [1] and are considered to be a rich source of essential oils-the metabolites, synthesized by plants for specific functions, using various secondary metabolic pathways. Humans have learned to use these metabolites since antiquity for food preservation, flavoring, and as medicine. The basil essential oils are usually extracted from the leaves and flowering tops of basil plants. Through the centuries basil was cultivated for culinary and medicinal purposes

\footnotetext{
* Correspondence: Trchounian@ysu.am

2Department of Biochemistry, Microbiology \& Biotechnology, Biology Faculty, Yerevan State University, 1 A. Manoogian Str., 0025 Yerevan, Armenia Full list of author information is available at the end of the article
}

in many countries, which created a great diversity of species within the Ocimum genus: the genus Ocimum comprises more than 150 species and is considered as one of the largest genera of the Lamiaceae family.

It is known, that different cultivars of basil have the genetic ability to generate and keep different sets of chemical compounds. This ability leads to a great variety of chemotypes within the same basil species. According to some investigations [2], the essential oils distilled from various basil cultivars can contain alcohols (linalool), oxides (1,8-cineole), phenols (eugenol, methyl eugenol, methyl isoeugenol, thymol), esters (methyl cinnamate), aldehydes (citral), and camphor. The 1,8-cineole, methyl cinnamate, methyl chavicol, and linalool are constituents responsible for the distinct aroma of basil plants [3]. 
Lawrence [4] named four major chemotypes of basil: methyl chavicol-rich, linalool-rich, methyl eugenol-rich, and methyl cinnamate-rich. Both methyl chavicol and methyl eugenol are phenylpropanoids produced by shikimic acid pathway and are reported to be toxic to insects and microbes. Linalool is a terpenoid produced by mevalonic acid pathway and known to possess antioxidant and antimicrobial activity [5]. Methyl cinnamate is the methyl ester of cinnamic acid. It is found naturally in many aromatic plants, including fruits like strawberry and is known to attract pollinators. According to Marotti et al. [6] the European basils are mostly of linalool and methyl chavicol types, whereas tropical basils have methyl cinnamate as their major constituent. Basils of methyl eugenol chemotype could be found growing in North Africa, Eastern Europe, and parts of Asia [7].

Numerous papers have been published on the antimicrobial and antioxidant properties of basil essential oils and its constituents. Koeduka et al. [8] and Zabka et al. [9] reported the antimicrobial activity of eugenol with analgesic properties for humans. Liu et al. [5] investigated the antioxidant and antimicrobial activity of linalool and geraniol. While Soković et al. [10] and Huang et al. [11] investigated the usage of linalool, methyl chavicol, and thymol for skin protection against all sources of environmental skin aggressors and treatment of various dermatological disorders.

Since the chemical composition (chemotype) and biological activity of essential oils distilled from the plants belonging to the same species may vary significantly, depending on the variety of cultivars, environment, elevation and cultivation methods, it is interesting to study the essential oils obtained from the different kinds of basil grown in Armenia, in similar conditions, at a significant elevation (1600 $\mathrm{m}$ above sea level).

In the present study the comparative analysis of the chemical composition and biological activities of essential oils distilled from three varieties of basil, O. basilicum var. purpureum, O. basilicum var. thyrsiflora, and O. x citriodorum, was carried out. The plants under investigation were grown in the same soil, at the same elevation, and under the same climatic conditions. The first two cultivars were varieties of $O$. basilicum species, or Sweet basil, and the third one, the Lemon basil $(O . x$ citriodorum) was a hybrid between $O$. basilicum and $O$. americanum. The purpose of this paper was also to study the biological activities of given oils and to evaluate their potential using in food industry, cosmetics and medicine.

\section{Methods}

\section{Plant material}

The three basil cultivars $(O$. basilicum var. purpureum, $O$. basilicum var. thyrsiflora, and O. x citriodorum) were grown from the seeds sown in the greenhouse, with subsequent transplantation of the seedlings to the same field, in the Kotayk Region of Armenia, where they have been growing side by side, at an elevation of $1600 \mathrm{~m}$ above the sea level. Plant materials were collected during blossoming period (July-August, 2014). The plant materials were identified at the Institute of Botany, National Academy of Sciences of Armenia, Yerevan (Armenia). The plants were not included in the herbarium as there were cultivated species and not typical for the flora of Armenia. The samples of basil cultivars are available at the Department of Microbiology \& Plants and Microbes Biotechnology, Biology Faculty, Yerevan State University, Yerevan, Armenia.

\section{Essential oil extraction}

Essential oils were extracted from air dried plant material (aerial parts only) by hydro-distillation, using a Clevengertype apparatus and lasted $3 \mathrm{~h}$. The distilled essential oils had been dehydrated with anhydrous sodium sulphate and stored at $4{ }^{\circ} \mathrm{C}$ in dark airtight bottles until further analysis [12].

\section{Determination of essential oil chemical composition}

The gas chromatography (GC) mass selective (MS) analysis of the essential oils was performed using a Hewlett-Packard 5890 Series II gas chromatograph, fitted with a fused silica HP - 5MS capillary column $(30 \mathrm{~m} \times 0.25 \mathrm{~mm}$, in thickness $0.25 \mu \mathrm{m})$. The oven temperature varied from $40-250{ }^{\circ} \mathrm{C}$ with the scanning rate of $3{ }^{\circ} \mathrm{C} / \mathrm{min}$. Helium (purity 5.6) was used as a carrier gas at a flow rate of $1 \mathrm{~mL} / \mathrm{min}$. The GC was equipped with Hewlett-Packard 5972 Series MS detector. The MS operating parameters were ionization voltage $70 \mathrm{eV}$ and ion source temperature $250{ }^{\circ} \mathrm{C}$. The diluted samples of essential oils $(1 / 100, \mathrm{v} / \mathrm{v}$ in HPLC methanol) of $1 \mu \mathrm{L}$ had been injected manually. To avoid overloading the GC column, the essential oils were diluted 1:100 (v/v) in methanol. The identification of peaks was tentatively carried out based on library search using National Institute of Standards and Technology (NIST)-2013. Relative Retention Index (RRI) was calculated for HP-5MS column. For RRI calculation a mixture of homologues $n$-alkanes (C9-C18) was used under the same chromatographic conditions as for analysis of the essential oils.

\section{Investigation of antimicrobial activity by agar diffusion method}

The antibacterial and antifungal activity of the essential oils was determined by the agar diffusion method [13]. This method was preferred over the dilution method because of low solubility of essential oils in water and in meat peptone broth. The following concentrations of 
essential oils were used: $150 ; 100 ; 50 ; 25 ; 12.5 ; 6.25 \mu \mathrm{L} /$ $\mathrm{mL}$; dimethyl sulfoxide (DMSO) was used as the solvent. The $100 \mu \mathrm{L}$ of each oil solution was introduced to the wells in the agar with test microorganisms. Different Grampositive (Bacillus subtilis WT-A, isolated from metal polluted soils of Kajaran, Armenia; Staphylococcus aureus MDC 5233 (Microbial Depository Center, Armbiotechnology Scientific and Production Center, Armenia; laboratory control strain) and Gram-negative (E. coli VKPM-M17 (Russian National Collection of Industrial Microorganisms at the Institute of Genetics and Selection of Industrial Microorganisms, Russia; laboratory control strain), Pseudomonas aeruginosa GRP3 (Soil and Water Research Institute, Iran) bacteria and ampicillin-resistant E. coli dhp $\alpha$-pUC18 were used. Bacterial cultures were grown on Mueller-Hinton agar. Ampicillin $(25 \mu \mathrm{g} / \mathrm{mL})$ as a positive control and DMSO as a negative control were used. The yeasts (Candida albicans WT-174 isolated from infected vaginal microbiota of hospitalized patients (clinical strain) and Debariomyces hansenii WT (French National Institute for Agricultural Research, France; laboratory control strain) were grown and maintained on Sabouraud-dextrose agar for $24 \mathrm{~h}$ at room temperature. As the positive control fluconazole $(25 \mu \mathrm{g} / \mathrm{mL})$ was used. Data were expressed in minimal inhibitory concentrations (MIC) values.

The selected pieces of nutrient medium from the zones of microorganism growth absence were transferred to the nutrient medium corresponding to each microorganism and then they were incubated for 23 days at appropriate temperature to determine the bacteriostatic or bactericidal action of the oils. The action of oils is evaluated as bacteriostatic in case of renewed growth of test-microorganisms after the recultivation.

\section{Determination of radical scavenging activity}

Free radical scavenging ability of the essential oils was tested using ethanol solution of 1,1-diphenyl-2-picrylhydrazyl (DPPH) [14]. Catechin was used as a positive reference. Sample solution contained $125 \mu \mathrm{L}(1 \mathrm{mM}) \mathrm{DPPH}, 375 \mu \mathrm{L}$ ethanol and $500 \mu \mathrm{L}$ of test-solution (essential oils or catechin with different concentrations). In the control solution the test-solution was replaced by ethanol. The absorbance was measured at the wavelength of $514 \mathrm{~nm}$.

The radical scavenging activity was calculated using the following formula: Radical scavenging activity (\%)= Ac-As/Ac $\times 100$, where Ac is absorbance of control (DPPH without the addition of test solution), and Asthe absorbance of the sample.

$\mathrm{IC}_{50}$ calculated denote the concentration of investigated samples required to decrease the DPPH absorbance at $514 \mathrm{~nm}$ by $50 \%$.

\section{Tyrosinase inhibition colorimetric assay}

Tyrosinase inhibition colorimetric assay was carried out according to the method, as described $[15,16]$. Each essential oil was dissolved in DMSO to obtain concentration of $20 \mathrm{mg} / \mathrm{mL}$. These stock solutions were diluted to $600 \mu \mathrm{g} / \mathrm{mL}$ concentration in $50 \mathrm{mM}$ potassium phosphate buffer ( $\mathrm{pH}$ 6.5). Arbutin acid was prepared in similar way and used as positive control. $700 \mu \mathrm{L}$ of each sample solution or positive control were combined with $300 \mu \mathrm{L}$ of mushroom tyrosinase (333 Unit per $\mathrm{mL}$ in phosphate buffer, $\mathrm{pH} 6.5)$. After incubation at $20-22{ }^{\circ} \mathrm{C}$ for $5 \mathrm{~min}, 1100 \mu \mathrm{L}$ tyrosine $(2 \mathrm{mM})$ were added to each well. Plates were incubated at room temperature for $30 \mathrm{~min}$ and the absorbance was measured at the wavelength of $492 \mathrm{~nm}$ using the spectrophotometer Genesys $10 \mathrm{~S}$ UV-vis (Thermo Scientific, USA). Percent inhibition of tyrosinase activity was calculated according to the formula: inhibition $(\%)=100-\left(\mathrm{W}_{\text {sample }} / \mathrm{W}_{\text {blank }}\right) \times 100$, where W is absorbance at $492 \mathrm{~nm}$. W blank is absorbance of control reaction (containing all reagents without test compound).

\section{Statistical analysis}

Experimental data $(n=4)$ were expressed as means with standard errors. The latter did not exceed 3\% (if not indicated). The validity of differences between experimental and appropriate control data were evaluated by Student's criteria (P) using Microsoft Excel 2010 with the help of $T$ test function; $P<0.05$ (if not indicated).

\section{Results}

Determination of chemical composition of essential oils

The results from the quantitative and qualitative analysis of essential oils constituents are presented in Table 1: the average yield of the essential oils was $0.2 \%$. More than 40 compounds were isolated, detected and most of them identified for each essential oil sample. The dominant components were identified to be linalool, methyl chavicol, citral and nerol.

According to the data obtained, O. basilicum var. purpureum contains $57.3 \%$ methyl chavicol, with the second largest component being linalool (18\%). This places the given variety of $O$. basilicum into methyl chavicol-rich chemotype. O. basilicum var. thyrsiflora belongs to linalool-rich chemotype, with concentrations of linalool and methyl chavicol being 68 and 20\% respectively. These data are in a good accordance with the results reported by Sishu et al. [17]. For the essential oil from $O . \times$ citriodorum species the predominant constituents were identified to be citral (21\%) and nerol (23\%), therefore it could not be classified as belonging to any of the chemotypes mentioned above, but will rather form its own, nerol-rich chemotype. 
Table 1 Chemical composition of essential oils of Ocimum basilicum var.purpureum, Ocium basilicum var. thyrsiflora, Ocimum citriodorum

\begin{tabular}{|c|c|c|c|c|}
\hline Chemical components & Relative Retention Index ${ }^{a}$ & O. basilicum var. purpureum, $\%^{\mathrm{b}}$ & O. basilicum var. thyrsiflora, $\%$ & O. x citriodorum, $\%$ \\
\hline 1-octen-3-ol & 979 & 0,2 & - & 0,1 \\
\hline 1-8- Cineole & 1035 & 1.40 & 3.50 & - \\
\hline (Z) - $\beta$-Ocimene & 1058 & - & - & 0.24 \\
\hline Y-Terpinene & 1078 & - & - & 0.22 \\
\hline Fenhone & 1089 & - & - & 0.32 \\
\hline Linalool & 1100 & 18.00 & 68.00 & 9.42 \\
\hline Camphor & 1146 & 1.30 & 1.35 & - \\
\hline a - Terpineol & 1181 & - & - & 0.62 \\
\hline Methyl chavicol & 1203 & 57.3 & 20.00 & 9.45 \\
\hline Nerol & 1231 & - & - & 23.00 \\
\hline Neral & 1244 & - & - & 4.93 \\
\hline Geraniol & 1259 & & & 5.20 \\
\hline Geranial & 1274 & - & - & 15.77 \\
\hline Bornyl acetate & 1291 & 0.13 & - & - \\
\hline Neryl acetate & 1321 & - & - & 0.65 \\
\hline Methyl cinnamate & 1338 & - & - & 0.49 \\
\hline$\beta$-Elemene & 1387 & 3.62 & 0.67 & 0.53 \\
\hline$\beta$-Caryophyllene & 1419 & 1.72 & - & 7.80 \\
\hline$\beta$-Copaene & 1428 & 0.28 & - & 0.56 \\
\hline trans-a-Bergamotene & 1433 & 4.34 & 1.34 & 3.52 \\
\hline a-Humulene & 1455 & 0.55 & 0.28 & 1.52 \\
\hline cis- $\beta$-Farnesene & 1472 & 0.31 & - & 0.48 \\
\hline Germacrene d & 1482 & 0.68 & 0.17 & - \\
\hline$\beta$-Cubebene & 1497 & - & 0.75 & 2.26 \\
\hline a-Bulnesene & 1502 & 1.39 & 0.68 & 0.47 \\
\hline a-Amorphen & 1510 & 1.54 & 0.69 & - \\
\hline$\delta$-Cadinene & 1518 & - & - & 0.38 \\
\hline Aromadendrene & 1529 & 1.67 & 0.28 & - \\
\hline Spathulenol & 1544 & 0.68 & - & - \\
\hline Caryophyllene oxide & 1550 & 0.57 & - & - \\
\hline a-Bisabolene & 1561 & - & - & 2.29 \\
\hline$\beta$ - Bisabolenene & 1572 & - & - & 8.31 \\
\hline a-Bisabolol & 1642 & - & - & 0.29 \\
\hline
\end{tabular}

for HP-5 capillary column

$\mathrm{b}_{\%}$ : Calculated from MIC data

The data on $O$. $\mathrm{x}$ citriodorum are somewhat consistent with the similar results published by CarovićStanko et al. [18] on essential oil distilled from the plant of the same species, except for the fact that there were more than 45 constituents of $O$. x citriodorum essential oil identified in the present study, as opposed to 20 components identified by CarovićStanko et al. [18].

\section{Antimicrobial activity of essential oils}

The present investigation revealed that Gram-positive bacteria tested were more sensitive to all three essential oils than Gram-negative bacteria (Fig. 1). Such tendency is also observed by other authors [19]. The essential oil of $O . \mathrm{x}$ citriodorum was quite active against $B$. subtilis and St. aureus, with the MIC of $3.125 \mu \mathrm{L} / \mathrm{mL}$. The same $\mathrm{MIC}$ was recorded for the essential oil of O. basilicum 


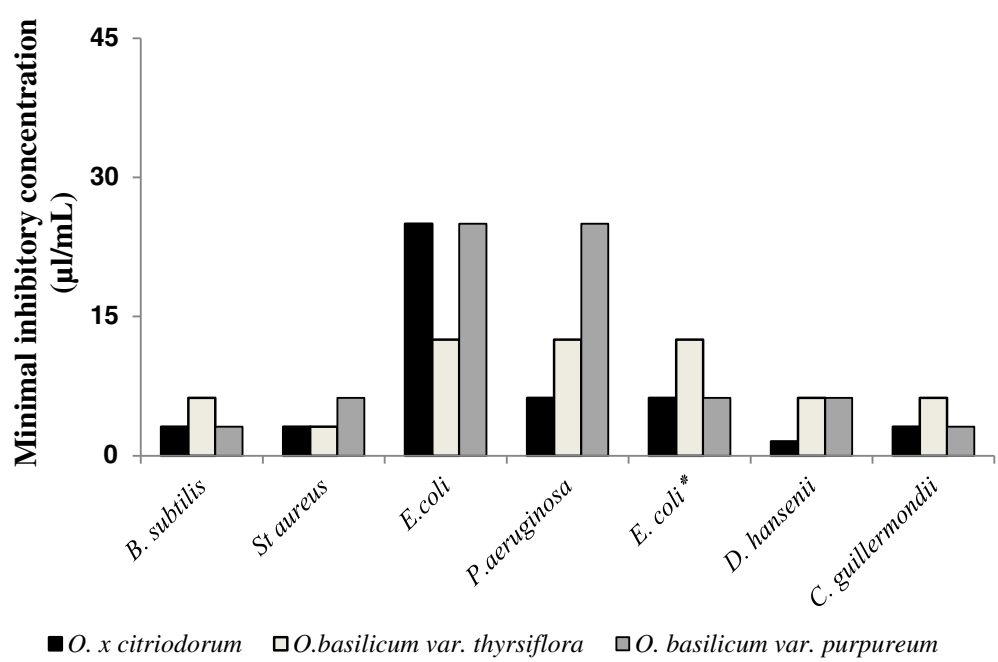

Fig. 1 The minimal inhibitory concentrations (MICS) of O. citriodorum, O. basilicum var. thyrsiflora and O. basilicum var. purpureum essential oils on selected Gram-positive, Gram-negative bacteria and fungi. Antibiotic-resistant E. coli dhpa-pUC18 strain was used. For bacteria and fungi strains and other details, see Methods. *Antibiotic-resistant E. coli dhpa-pUC18 strain

var. thyrsiflora against St. aureus, and O. basilicum var. purpureum essential oil against $B$. subtilis. The MIC of O. basilicum var. thyrsiflora essential oil against B. subtilis and MIC of O. basilicum var. purpureum against St. aureus were twice as high, $6.25 \mu \mathrm{L} / \mathrm{mL}$. The ampicillinresistant $E$. coli bacteria also displayed sensitivity against the essential oils tested: thus the MIC values of $O . x$ citriodorum and $O$. basilicum var. purpureum against those bacteria were $6.25 \mu \mathrm{L} / \mathrm{mL}$, while $O$. basilicum var. thyrsiflora displayed MIC of $12.5 \mu \mathrm{L} / \mathrm{mL}$. The action of the essential oils on the all bacteria in this study was evaluated as bactericidal.
All three essential oils have also displayed high antifungal activity, with $O$. x citriodorum being the strongest antifungal amongst them: MIC of $O$. x citriodorum against $D$. hansenii and C. guillermondii were 1.56 and $3.125 \mu \mathrm{L} / \mathrm{mL}$, respectively (see Fig. 1 ).

\section{Radical scavenging activity}

The results of radical DPPH assay for of O. x citriodorum, O. basilicum var.purpureum, O. basilicum var. thyrsiflora essential oils are shown on Fig. 2 . The highest antioxidant activity was demonstrated by $O$. basilicum var. thyrsiflora essential oil: $\mathrm{IC}_{50}$ value for it was equal

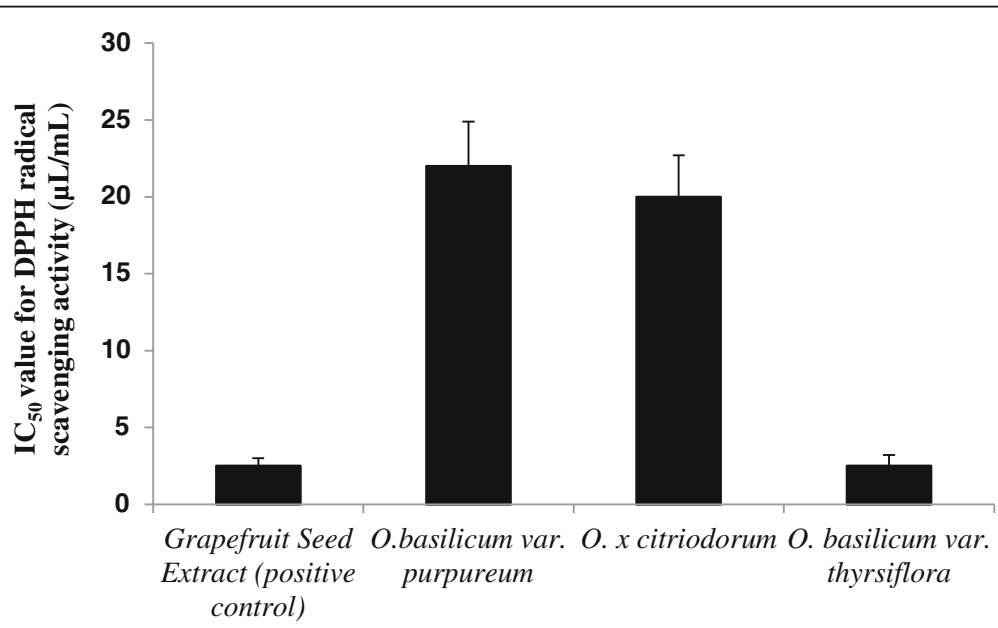

Fig. $2 \mid I_{50}$ values of antiradical activity of $O$. citriodorum, $O$. basilicum var. thyrsiflora and $O$. basilicum var. purpureum essential oils. For details, see Methods 
to the standardized Grapefruit Seed Extract which was used as a control sample $(2.5 \mu \mathrm{L} / \mathrm{mL})$. The antiradical activity for the other two basil species was lower: $\mathrm{IC}_{50}$ value for $O$. x citriodorum essential oil was $20 \mu \mathrm{L} / \mathrm{mL}$ and for O. basilicum var. purpureum was $22 \mu \mathrm{L} / \mathrm{mL}$. These results were somewhat unexpected, since usually the oils with higher phenolic content are the ones exhibiting higher radical scavenging abilities, whereas in our case the highest antioxidant properties were displayed by the cultivar with the highest linalool (terpene alcohol) content.

\section{Tyrosinase inhibition activity}

The enzyme tyrosinase inhibition abilities of all three oils were also assessed as a part of our efforts to find a natural treatment for hyper-pigmentation skin disorder. The values for tyrosinase inhibitory activity of $O$. basilicum var. thyrsiflora, $O$. basilicum var. purpureum and $O$. $\mathrm{x}$ citriodorum essential oils and arbutin acid (positive control) were calculated to be $20.1 \pm 1.4 \%$; $11.5 \pm 0.3 \%$; $17.4 \pm$ $0.9 \%$ and $81.5 \pm 2.6 \%$, respectively (Fig. 3).

\section{Discussion}

Under the experimental conditions of the present study it was revealed that the dominant constituent for $O$. basilicum var. purpureum is methyl chavicol (estragol), whereas the major component for the other variety of the same species, O. basilicum var. thyrsiflora is linalool. At the same time, the chemical composition of O. citriodorium hybrid plant differed substantially from the first two basil varieties: it had significant aldehyde content, represented by citral, with another prevalent constituent being nerol (monoterpene alcohol). Neither citral nor nerol was detected in the two other species of Ocimum (see Table 1). We observed that the essential oil from $O$. citriodorum species displayed the highest antimicrobial activity against the most of microorganisms tested. The experiments showed that essential oils from all three varieties of basil can significantly inhibit the growth of ampicillin-resistant strain of $E$. coli bacteria. It is interesting to notice, that the observed antibacterial activities of the essential oils from O. citriodorum and O. basilicum var. purpureum against $E$. coli where much higher in case of ampicillin-resistant strain than in the case of a non-resistant one. At the same time, the essential oil from $O$. thyrsiflora cultivar displayed the same, relatively high antibacterial activity in both cases (see Fig. 1).

The essential oils from all three basil cultivars tested showed high inhibition activities against fungi and high radical scavenging activity. Among the three, the essential oil from the O. thyrsiflora variety displayed the highest ability to neutralize free radicals and showed results similar to the positive control.

The essential oils from all three varieties exhibited some tyrosinase inhibitory activity, although it wasn't particularly high.

The essential oils from both $O$. citriodorum and $O$. thyrsiflora varieties of basil show high inhibition rates against $S$. aureus bacteria, which makes it possible to consider using these oils as active natural ingredients for the treatment of skin irritations, since $S$. aureus is extremeley common on the skin of patients with certain dermatological diseases [20], and it is often considered to be a major culprit in causing skin irritation and soft

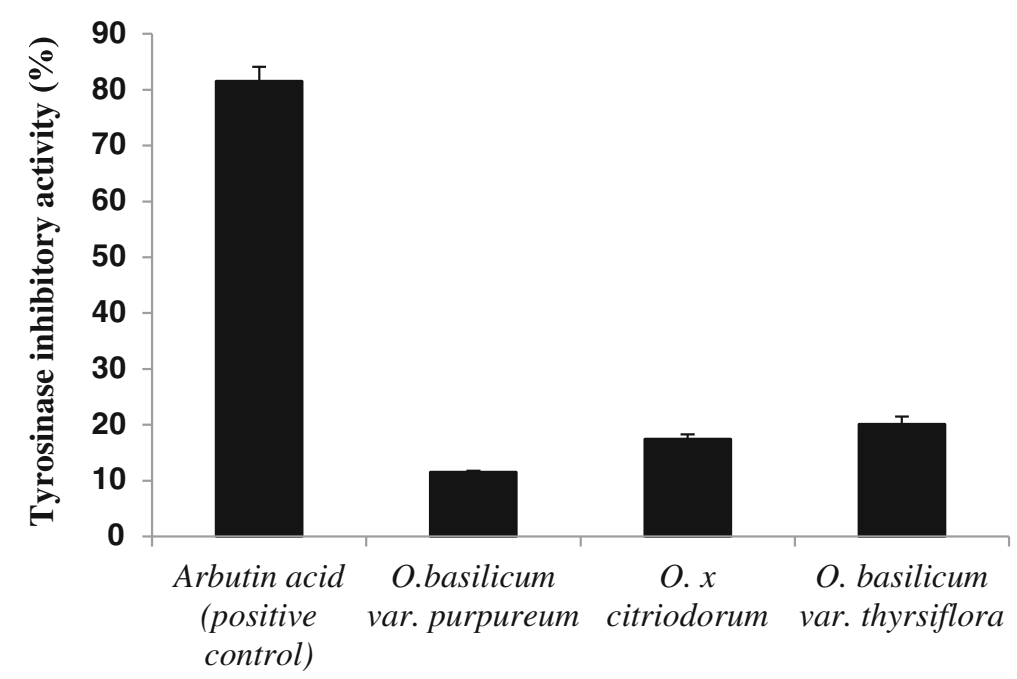

Fig. 3 The tyrosinase inhibitory activity of $O$. basilicum var. thyrsiflora and O. basilicum var. purpureum, O. citriodorum essential oils. For details, see Methods 
tissue infections [21]. At the same time, the combination of very strong antioxidant properties with some tyrosinase inhibition abilities makes the essential oil of $O$. thyrsiflora a good candidate to be used as a multifunctional cosmetic active in various cosmetic formulas, namely as an antioxidant with some additional skin brightening properties.

\section{Conclusions}

The qualitative and quantitative composition of the three essential oils of three basil cultivars (O. basilicum var. thyrsiflora, $O$. basilicum var. purpureum and $O . \mathrm{x}$ citriodorum), cultivated in Armenia, was quite different: O. basilicum var. purpureum essential oil contained 57.3\% methyl-chavicol (estragol); O. basilicum var. thyrsiflora oil had $68.0 \%$ linalool, and the main constituents of O. x citriodorum oil were nerol (23.0\%) and citral (20.7\%). The presence of thyrosinase inhibitory activity is enhances the pharmacological value of these oils. They had also high antioxidant, antibacterial and antifungal activity and could be used as good sources of natural antimicrobial and antioxidant agents, with possible application in food industry, cosmetics or medicine.

\section{Abbreviations}

DMSO: Dimethyl sulfoxide; DPPH: 1,1-diphenyl-2-picrylhydrazyl; GS: Gas chromatography; MIC: Minimal inhibitory concentration; MS: Mass selective; NIST: National Institute of Standards and Technology; P: Student's criteria; RRI: Relative retention index

\section{Acknowledgement}

This study was done in the frame of the cooperation with Nairian CJSC (Armenia).

\section{Funding}

This study was done in the frame of Basic research support by State Committee of Science, Ministry of Education and Science of Armenia, to Yerevan State University.

\section{Availability of data and materials}

The plant materials and methods used (see hereafter) were available upon request. All data obtained have been included into the manuscript.

\begin{tabular}{ll}
\hline $\begin{array}{l}\text { Data scope and type } \\
\text { Identification of chemical } \\
\text { substance peaks }\end{array}$ & $\begin{array}{l}\text { Database } \\
\text { National Institute of Standards and } \\
\text { Technology (NIST)-11-2013 } \\
\text { (http://www.nist.gov/srd/upload/ } \\
\text { NIST1a11Ver2-OMan.pdf) }\end{array}$ \\
$\begin{array}{ll}\text { Protocols of testing of } \\
\text { antimicrobial activities by } \\
\text { agar-diffusion method }\end{array}$ & $\begin{array}{l}\text { USA } \\
\text { (http://ncipd.org/Control/images/ }\end{array}$ \\
& NCIPD_docs/CLSI_M100-S24.pdf) \\
Protocols for determination \\
of antiradical activity
\end{tabular}$\quad \begin{aligned} & \text { International Union of Pure and Applied } \\
& \text { Chemistry, Analytical Chemistry Division } \\
& \text { (http://www.bashanfoundation.org/shela/ } \\
& \text { shelaiupac.pdf) }\end{aligned}$

\section{Author's contribution}

AA collected plant material, obtained essential oils and identified chemical structure of essential oil components; AM contributed to manuscript preparation and improved English; MP identified plants, developed the methods and contributed to manuscript preparation; NS developed the methods and prepared the manuscript; $A B$ tested the biological activities of essential oils and analyzed data; SA obtained essential oils and provided chemical analyses; AT supervised the study and edited the manuscript. All authors have read and approved the manuscript.

\section{Competing of interest}

The authors declare no commercial, financial or any other conflict of interest.

\section{Consent for publication \\ Not applicable.}

\section{Ethics approval and consent to participate}

Not applicable.

\section{Author details}

${ }^{1}$ Nairian CJSC, Khorenatsi 15, Yerevan, Armenia. ${ }^{2}$ Department of Biochemistry, Microbiology \& Biotechnology, Biology Faculty, Yerevan State University, 1 A. Manoogian Str., 0025 Yerevan, Armenia.

Received: 16 September 2016 Accepted: 14 January 2017

Published online: 19 January 2017

\section{References}

1. Paton A, Harley MR, Harley MM. Ocimum: an overview of classification and relationships". Published by license under the Harwood Academic Publishers imprint, part of the Gordon and Breach Publishing Group. Amsterdam: OPA (Overseas Publishers Association) N.V.; 1999;38.

2. Kruger $\mathrm{H}$, Wetzel SB, Zeiger B. The chemical variability of Ocimum species. J Herbs Spices Med Plants. 2002;9:335-44.

3. Klimankova E, Holadova K, Hajslova J, Cajka T, Poustka J, Koudela M. Aroma profile of five basil (Ocimum basilicum L.) cultivars grown under conventional and organic conditions. Food Chem. 2008;107:464-72.

4. Lawrence BM. A further examination of the variation of Ocimum basilicum L. In: Lawrence BM, Mookerjee BD, Willis BJ, editors. Flavors and fragrances: A world perspective. Amsterdam: Elsevier Sci. Publ. B.V; 1988. p. 161-70.

5. Liu K, Cheng Q, Liu Y, Zhou X, Wang X. Isolation and biological activities of decanal, linalool, valencene, and octanal from sweet orange oil. J Food Sci. 2012;77:1156-61.

6. Marotti M, Piccaglia R, Giovanelli E. Differences in essential oil composition of basil (Ocimum basilicum L.) Italian cultivars related to morphological characteristics. J Agric Food Chem. 1996;44:3926-9.

7. Joshi RK. Chemical composition and antimicrobial activity of the essential oil of Ocimum basilicum L. (sweet basil) from Western Ghats of North West Karnataka, India. Anc Sci Life. 2014;33:151-6.

8. Koeduca T, Fridman E, Gang DR, Vassão DG, Kackson BL, Kish CM, Orlova I, Spassova SM, Lewis NG, Noel JP, Baiga TJ, Dudareva N, Pichersky E. Eugenol and isoeugenol, characteriastic aromatic constituents of spices, are biosynthesized via reduction of a conifer alcohol ester. Proc Natl Acad Sci U S A. 2006;103:10128-33.

9. Zabka M, Pavela R, Prokinova E. Antifungal activity and chemical composition of twenty essential oils against significant indoor and outdoor toxigenic and aeroallergenic fungi. Chemosphere. 2014;112:443-8.

10. Soković M, Marin PD, Brkić D. L. van Griensven. Chemical Composition and antibacterial activity of essential oils of ten aromatic plants against human pathogenic bacteria. Food Glob Sci. 2007;1:220-6.

11. Huang HC, Ho YC, Lim JM, Chang TY, Ho CL, Chang TM. Investigation of the anti-melanogenic and antioxidant characteristics of Eucalyptus camaldulensis flower essential oil and determination of its chemical composition. Int J Mol Sci. 2015;16:10470-90

12. Council of Europe. European Pharmacopeia. 5th ed. Strasbourg: European Council, European Directorate for the Quality of The Medicines (EDQM). 2005;1.

13. Patel JB, Cockerill FR, Alder J, Bradford PA, Eliopoulos GM, Hardy DJ, Hindler JA, Jenkins SG, Lewis JS, Miller LA, Powell M, Swenson JM, Traczewski MM, Turnidge JD, Weinstein MP, Zimmer BL. Performance Standards for Antimicrobial Susceptibility Testing; Twenty-Fourth Informational 
Supplement. CLSI document M100-S24. Wayne, PA: Clinical and Laboratory Standards Institute; 2014.

14. Apak R, Gorinstein S, Böhm V, Schaich KM, Özyürek M, Güçlü K. Methods of measurement and evaluation of natural antioxidant capacity/activity (IUPAC Technical Report). Pure Appl Chem. 2013;85:957-98.

15. Al-Mamary M, Abdewahab SI, Al-Ghalibi S, Al- Ghasani E. The antioxidant and tyrosinase inhibitory activities of some essential oils obtained from aromatic plants grown and used in Yemen. Sci Res Essays. 2011;6:6840-5.

16. Wang YH, Avonto C, Avula B, Wang M, Khan IA. Quantitative determination of a-arbutin, $\beta$-arbutin, kojic acid, nicotinamide, hydroquinone, resorcinol, 4methoxyphenol, 4-ethoxyphenol, and ascorbic acid from skin whitening products by HPLC-UV. J AOAC. 2015;98:5-12.

17. Sishu R, Tadesse S, Bucar F, Asres K. Composition and the antioxidant activity of the essential oils of Ocimum basilicum var. thyrsiflorum and Ocimum americanum. Int J Essent Oil Ther. 2010:4:64-8.

18. Carović-Stanko K, Orlić S, Politeo O, Strikić F, Kolak I, Milos M, Satovic Z. Composition and antibacterial activities of essential oils of seven Ocimum taxa. Food Chem. 2010;119:196-201.

19. Sarrazin SLF, Oliviera RB, Barata LES, Mourao RHV. Chemical composition and antimicrobial activity of the essential oil of Lippia grandis Schauer (Verbenaceae) from the western Amazon. Food Chem. 2012;34:1474-8.

20. Baron S. Medical Microbiology. 4th edition, Chapter 6, University of Texas, 1996

21. Kong HH, Oh J, Deming C, Conlan S, Grice EA, Beatson MA, Nomicos E, Polley EC, Komarow HD, Murray PR, Turner ML. Temporal shifts in the skin microbiome associated with disease flares and treatment in children with atopic dermatitis. Genome Res. 2012;22:850-9.

\section{Submit your next manuscript to BioMed Central and we will help you at every step:}

- We accept pre-submission inquiries

- Our selector tool helps you to find the most relevant journal

- We provide round the clock customer support

- Convenient online submission

- Thorough peer review

- Inclusion in PubMed and all major indexing services

- Maximum visibility for your research

Submit your manuscript at www.biomedcentral.com/submit
Biomed Central 